\title{
A wireless local area network employing distributed radio bridges*
}

\author{
Victor C.M. Leung and Andrew W.Y. Au \\ Department of Electrical Engineering, The University of British Columbia, 2356 Main Mall, Vancouver, B. C., Canada V6T $1 Z 4$
}

\begin{abstract}
This paper presents a novel distributed wireless local area network (WLAN) architecture, where each wireless terminal (WT) accesses a backbone local area network (LAN) segment via multiple radio bridges (RB's). We introduce a self-learning routing algorithm for the RB's, which automatically adapts to changes in terminal locations, and prevents multiple copies of each data frame from being forwarded over the backbone LAN segment. The distributed WLAN architecture eases the management of network topological changes and terminal mobility, compared to centralized cellular architectures. We consider the use of direct-sequence spread-spectrum (DS/SS) signaling and the slotted Aloha medium access control (MAC) protocol over the wireless links, with multiple uplink receivers and downlink transmitters at the RB's for each MAC frame. Simulation results for the uplink show that the multi-receiver site diversity and the capture effect of DS/SS signaling effectively combat multipath and multiaccess interference, resulting in high throughput capacity and stable operation for the channel. Under overload traffic, the system is able to maintain a high level of throughput with bounded delays. It is shown that the use of multiple receiver reduces the access fairness problem for WT's at different locations caused by the near-far effect.
\end{abstract}

\section{Introduction}

WLAN's employ wireless communications for interconnecting wireless terminals (WT's) among themselves and/or to wire-based LAN's. Wireless access facilitates flexible location of terminals, providing connectivity to mobile terminals and avoiding re-wiring when fixed terminals are relocated. Where a LAN needs to be reconfigured from time to time, the use of wireless access can give tremendous savings in the life time cost of the system, as it largely eliminates the cabling costs associated with network reconfigurations, which can be substantial [1]. A WLAN usually incorporates a medium access control (MAC) protocol [2] to enable multiple WT's to share the same radio or optical wireless channel. Where a backbone LAN is employed for extending the connectivity of a WLAN, a bridging algorithm [3] is required at the points of interconnection, referred to as the radio bridges (RB's) in this paper, to harmonize the MAC protocols for the wireless channel and the LAN, and to facilitate proper forwarding of the data frames.

To provide coverage in a large building using lowpower radio transmissions, one approach is to divide the building area into cells, as in a cellular mobile telephone system, and connect these cells through a backbone LAN, as illustrated in Fig. 1. Different radio channels are assigned to adjacent cells to minimize interference. Each radio channel is re-used at cells separated by sev-

\footnotetext{
* Parts of this paper were presented at IEEE Pacific Rim Conference, Victoria, B.C., May 1993, and IEEE International Conference on Selected Topics in Wireless Communications, Vancouver, B.C., June 1992. This work was partially supported by the Canadian Natural Sciences and Engineering Research Council under grant OGP0044286 .
}

eral cell diameters. This is a centralized architecture since each WT is covered by a single cell. In each cell, all WT's communicate over the same radio channel using a specific MAC protocol, and access the backbone LAN via a single $\mathrm{RB}$. This is the approach of Motorola's Wireless In-building Network [4,5], which employs FDMA between cells and TDMA within each cell. This centralized architecture has the advantage that the MAC protocol employed within each cell could be optimized with respect to the traffic in the cell. Another advantage is that internetworking between the WLAN and the backbone LAN is simplified by the application of the selflearning bridging algorithm for LAN bridges [3]. However, configuration and management of the centralized network, particularly with respect to changes in network coverage and mobility of WT's, are complicated by the need of frequency coordination between cells and handing off WT's moving across cell boundaries. The spread-

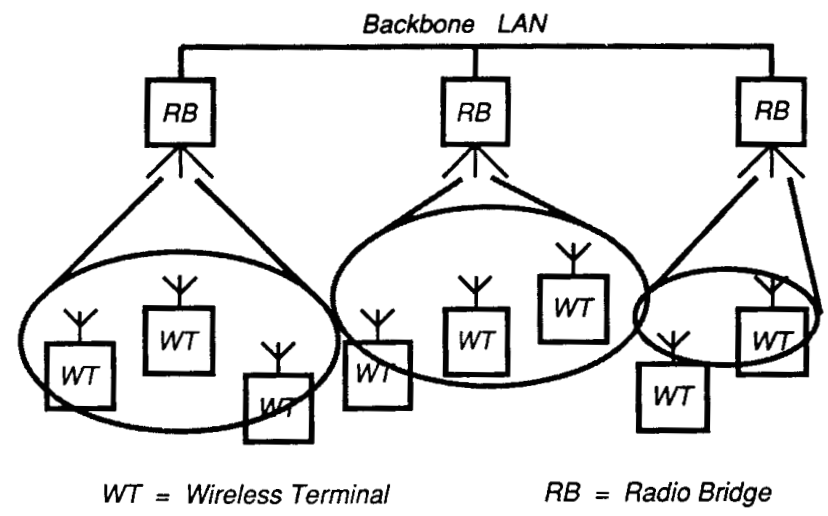

Fig. 1. Cellular architecture for WLAN, with centralized access in each cell. 
spectrum CDMA approach employs code orthogonality rather than frequency and time orthogonality for separation of user traffic between cells and within each cell, and substantially eases the frequency coordination and handoff problems [6,7]. However, the complex multiple correlator receiver structure required to support simultaneous multi-user access at each RB is very costly, and seems more appropriate to the circuit-switched voice traffic typical in cellular networks than the packetswitched data traffic in a WLAN.

This paper presents a new approach for WLAN's, employing a distributed architecture and slotted Aloha access with DS / SS signaling over the wireless channel. It retains many of the advantages of CDMA, but with simpler and hence less expensive receivers at the RB's, since all WT's employ the same code sequence for transmissions to all RB's, and vice versa. The network architecture is elaborated in section 2. The distributed WLAN architecture is similar to that considered in [8], and is based upon using the same radio channel over the entire coverage area, with multiple RB's interconnecting each WT to a backbone LAN segment, providing macroscopic site diversity for each wireless transmission between each WT and the backbone LAN segment. This network architecture has also been considered for mobile data networks with multiple base sites [9]. The distributed architecture eliminates the need for frequency coordination, and greatly eases network configuration and management. In particular, terminal mobility is easily accommodated without using complicated handoff mechanisms as required in cellular architectures.

An internetworking problem unique to the distributed WLAN architecture is the possibility of RB's forwarding multiple copies of each WT originated frame onto the LAN segment. Whereas the internetworking scheme in [8] allows data frames received in error over the radio channel by the RB's to be forwarded over the LAN, we assume that only correctly received frames are forwarded, while incorrectly received frames are discarded. The bridging algorithm, specified in section 3 , is a modification of the self-learning bridging algorithm [3], and is capable of dynamically adapting to changing WT locations.

The radio link takes advantage of the capture effect of DS / SS signaling to overcome multipath and multiaccess interference, and is modeled in section 4. Multiaccess interference in the radio link results in the collision of frames transmitted by several users at the receiver. In conventional multiaccess models [2], all colliding frames are assumed to be destroyed, so that multiaccess interference severely limits the throughput efficiency of the channel. However, the capture effect enables one of several colliding frames to capture the receiver and be correctly received. This is possible due to the power differences between the interfering signals [10], or the use of DS / SS signaling [11]. Therefore the capture effect greatly increases the throughput efficiency of the channel in the presence of multiaccess interference. The performance of multi-receiver slotted Aloha access, taking into account of the capture effects, has been analyzed [12]. However, this analysis unrealistically assumed that each frame is received with equal power at all the receivers, and the capture effect is based only on power differences of the received signals [10]. We present a more accurate model for DS/SS signal capture, where the frame error rate of the signal successfully captured by the code synchronizer in a receiver is explicitly evaluated with respect to the effects of multipath and multiaccess interferences. We present simulation results for uplink channel throughput and delay in section 5, for WT's located in a closed room with different number of RB's. The results show that the system achieves a high channel throughput which approaches the ideal value of $100 \%$ for each $\mathrm{RB}$ receiver, while maintaining stable system operation, under heavy traffic condition. Concluding remarks are given in section 6 .

\section{Distributed WLAN architecture}

Fig. 2 illustrates the configuration of the proposed WLAN, with multiple WT's accessing a backbone LAN segment via distributed RB's. All WT's transmit over the same (uplink) radio channel to the RB's, and receive over a different (downlink) radio channel from the RB's. The transmissions in each radio channel employ DS/SS signaling with a common pseudonoise (PN) spreading code. In DS/SS signaling, the transmitter multiplies the data symbols with the PN code running at a much higher rate (the chip rate) than that of the data (the data rate), thus spreading the data bandwidth to a much wider transmission bandwidth. Typically, the chip rate equals $L$ times the data rate, where $L$ is the period of the PN code sequence. In the proposed system, at any time each receiver is capable of correlation reception of a single transmission only. The transmission which has been acquired by the receiver code synchronizer and is in

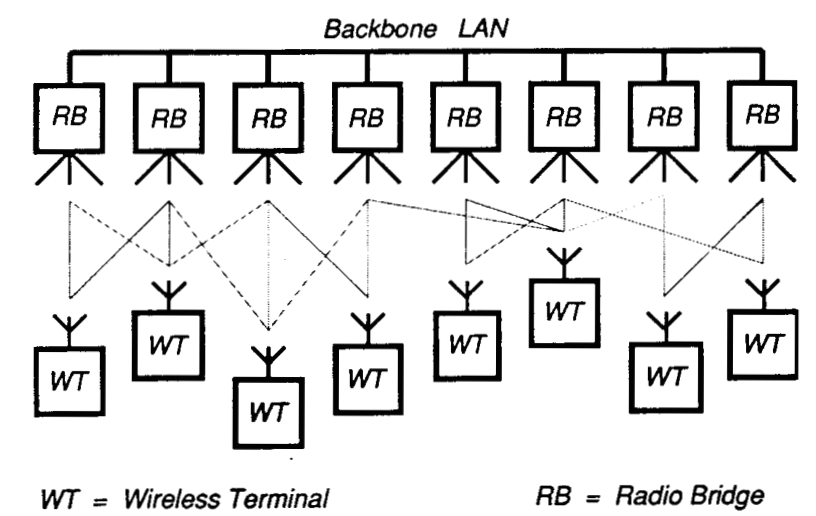

Fig. 2. Distributed architecture for WLAN, with access via multiple RB's. 
lock with the receiver generated code sequence is said to have captured the receiver. In this case, the receiver has a spread-spectrum processing gain of $G_{P}=L$, achieved in the process of compressing the bandwidth of the received signal to the data bandwidth [13]. In particular, multipath and multiaccess interference signals are attenuated by the processing gain when they are out of phase by more than 1 chip (duration of each PN code bit) with the signal to which the receiver is in synchronization. If the receiver acquires the strongest among several contending signals, then out of phase multipath and multiaccess interference signals are effectively rejected. Spread-spectrum signaling also reduces interference between different systems that might share the same frequency band. The processing gain reduces co-channel interference from other systems at the spread-spectrum receiver, while the wide-band signal with low spectral density transmitted by the spread-spectrum transmitter minimizes the interference power per unit bandwidth imparted on other systems. Therefore, with spread-spectrum signaling, it is feasible for WT's and RB's in a WLAN to operate within the emission level required for unlicensed transmitters, i.e., $1 \mathrm{~W}$ in the industrial, scientific and medical (ISM) bands (902-928 MHz, 2,4002,483.5 MHz, and 5,725-5,850 MHz [14]).

Each WT consists of a workstation equipped with a wireless transceiver, and employs the slotted Aloha MAC protocol to communicate with the RB's within its range. Each $\mathrm{RB}$ incorporates a processing unit which buffers and forwards incoming MAC frames according to the bridging algorithm described in the next section, a wireless transceiver for communications with WT's within its coverage area, and a LAN interface for communications with fixed terminals or other RB's over the LAN using the MAC protocol for the LAN. The locations of the mobile WT's are variable. We assume that sufficient number of RB's are attached to a common backbone LAN segment at fixed locations throughout the building to provide multiple-site radio coverage of all possible locations of the WT's. Multiple uplink/ downlink connections exist between each WT and the RB's within its range, providing receiver site diversity for the WT's transmissions, and transmitter site diversity for transmissions to the WT. The usable connections for each WT vary with time as changes occur in propagation environment and the WT's location. The site diversity enhances the probability of successful reception for each frame.

The proposed WLAN architecture lends itself to easy expansion of network capacity and reconfiguration of network topology. Adding WT's in the existing network coverage area is straight forward, with no more involvement of network management functions than is necessary for fixed terminals. However, the number of WT's within a certain area may be limited by the available network throughput capacity per unit area. Since the throughput available in a given area is limited by the cluster of RB's providing overlapping coverage of that area, the throughput may be increased (within certain limits) by reducing the coverage of each RB in that area, and increasing the number of RB's. This is similar to the idea of cell splitting in cellular networks. What it entails is the reduction of transmit power at the RB's and the covered WT's. It is also easy to expand the coverage area of the network. Initially a network would include only sufficient RB's to cover the current range of movements of WT's. Ideally the backbone LAN segment interconnecting the RB's would be installed to cover all building areas, whether RB's are currently attached or not. The coverage area of the network may then be expanded by simply attaching additional RB's to the backbone LAN segment to cover the new areas. Alternately, the backbone network may be extended to interconnect the additional RB's. Note that support of site diversity using the frame forwarding algorithm presented in the next section is limited to RB's connected to a common LAN segment not separated by bridges. In case the backbone network needs to be expanded via additional LAN segments interconnected by bridges, each LAN segment should interconnect a disjoint WLAN, such that the coverage areas of two RB's in different WLAN's attached to different LAN segments do not overlap.

\section{Bridging algorithm}

The bridging algorithm enables the distributed RB's connected to a common LAN segment to harmonize the MAC protocols for the wireless channel and the LAN, and to forward data frames to the proper network. Frame conversion is unnecessary if the MAC protocols utilize compatible frame formats, thus reducing the complexity of bridging. Frame forwarding is based on the use of a routing table and the execution of the frame forwarding algorithm in each RB.

\subsection{Self-learning routing}

A RB receives and processes all frames broadcasted over the LAN segment, but it only receives and processes frames broadcasted by those WT's that are within its radio range. The issue is to decide whether to forward or not to forward a frame received over the wireless channel onto the LAN, and vice versa. In general, all frames received over the uplink wireless channel must be forwarded to the LAN or rebroadcast over the downlink wireless channel. However, frames received over the LAN are forwarded via the downlink wireless channel only if the destination WT is within range of the RB, as determined by a routing table stored in the $\mathrm{RB}$. The routing table is built up using a self-learning process: whenever the RB receives a frame from a WT over the uplink wireless channel, the WT is identified as being reachable and its address is added to the routing table, if it is not 
already in the table. This assumes that the uplink and downlink connections between the WT and the RB are symmetrical. The routing table must be prevented from growing monotonically in size as existing WT's move out of range and new WT's move within range of the RB. In transparent LAN bridges, this is accomplished by periodically flushing the routing table and rebuilding it from scratch. Since each RB typically serves a relatively small WT population, a better method is to have a $\tau$ sec timer associated with each routing table entry. The timer is started whenever the $\mathrm{RB}$ receives a frame from the corresponding WT. When the timer expires, the WT's entry is deleted from the routing table. To maintain its entries in surrounding RB's when a WT is inactive for a prolonged period of time, the WT is required to send a "hello" frame to the RB's every $\tau$ sec when it has no data frame to transmit. The self-learning routing easily allows a WT to leave the WLAN associated with one LAN segment and join another WLAN associated with a different LAN segment.

\subsection{Frame forwarding algorithm}

Assuming each WT is within range of some RB's attached to a common LAN segment and transmits one data frame or "hello" frame at least once every $\tau$ sec, all WT's are therefore logically connected to the network through some RB's, i.e., their addresses are entered in the routing tables of these RB's. To take advantage of the inherent site diversity in the wireless channel, the frame forwarding algorithm allows all RB's within range of a WT to receive a frame from the WT, but only one to forward it over the common LAN segment connecting these RB's so as to conserve LAN bandwidth. On the other hand, several RB's within range of a WT may attempt to forward frames addressed to the WT. Delaying the downlink transmissions by a random delay of maximum 1-bit interval (the downlink randomization delay) enables the spread-spectrum receiver at the WT to capture the strongest transmission from these RB's, as discussed in the previous section. The frame forwarding algorithm executed by each RB is as follows:

1. Frame received from a WT over the uplink - The frame is stored in a buffer (the uplink buffer) for transmission over the LAN. If the destination address is also found in the RB's routing table, i.e., the destination is a WT within range of the $R B$, the frame is rebroadcast over the wireless channel after it has been successfully transmitted over the LAN.

2. Frame received over the LAN - The frame is compared with the current frame (if any) stored in the uplink buffer, which is then cleared if both frames are identical. In all cases, the frame is forwarded over the wireless channel after the downlink randomization delay if the destination address is found in the RB's routing table.
If the destination of a frame received at $\mathrm{RB}_{i}$ is a WT within its range, it may seems redundant in (1) above to also forward the frame over the LAN. However, (i) there exists other RB's which may be within range of the source WT but not the destination WT, and (ii) vice versa. In the case (i), other RB's will also attempt to forward the frame over the LAN, and the algorithm ensures that only one frame is forwarded. In case (ii), forwarding the frame over the LAN ensures that multiple RB's will transmit the frame to the destination WT. The reason for rebroadcasting the frame only after it has been successfully transmitted over the LAN is to ensure that $\mathrm{RB}_{i}$ only rebroadcasts the frame to the destination once, even though it may also have received the same frame from another RB over the LAN. The requirement for the $\mathrm{RB}$ to compare each frame received over the LAN with the frame in the uplink buffer may seem expensive in the amount of processing required. However, this comparison needs not be done on the entire frame for every frame received, but instead, it can be done on an incremental basis. Thus most frames would be rejected simply based on the comparison of the address fields. Since the RB has a definite need to perform address comparison on every frame received over the LAN against its routing table entry, the comparison with the uplink buffer represents only a small increment in the amount of processing required in the $\mathrm{RB}$.

The above frame forwarding algorithm is generally applicable for any standard MAC protocol employed by the LAN, such as CSMA/CD or token passing, which allows the transmission of one frame at a time over a LAN segment. Note that while a RB is receiving a frame from the LAN, it may not transmit on the LAN at the same time. The algorithm therefore guarantees that only one copy of a frame is transmitted over the LAN segment. With token passing, the first among many RB's buffering the same frame to capture a token will successfully forward the frame over the LAN segment while erasing the copies of this frame from the buffers of the other RB's. If CSMA/CD is employed, transmissions of the same frame by several RB's may collide with each other. However, the binary exponential backoff algorithm of CSMA/CD is capable of resolving the conflict, so that the first RB to successfully transmit over the LAN segment will also erase the multiple copies of the frame stored in the other RB's.

Note that if the RB's are distributed over several LAN segments connected by self-learning bridges, the duplication prevention property of the frame forwarding algorithm no longer holds, and it is not possible to ensure that a frame destined to a WT are forwarded over multiple RB's. Thus it is desirable to separate the RB's connected by individual LAN segments into nonoverlapping WLAN's. Mobility of WT's between these WLAN's is still supported, although the advantages of site diversity is lost during such movements. If this separation is not possible, some frame duplications may 
occur over the LAN resulting in inefficient utilization of the LAN bandwidth. However, due to the connectionless nature of the MAC layer service, such duplicates generally do not affect the operations of higher layer protocols as they are rejected by a higher layer connection-oriented protocol, e.g., TCP. Similarly, since the MAC layer does not guarantee frame delivery, loss of packets is also recovered by a connection-oriented protocol at a higher layer.

\section{Model of DS/SS signaling}

Here we present the model for the wireless channel, which forms the basis for the performance analysis of the uplink in the next section. In this model, DS/SS signaling with BPSK modulation is employed. All transmitters and receivers use a common spreading sequence of the class AO/LSE m-sequence (auto-optimal maximallength sequence with least sidelobe energy) [15] for its good periodic correlation property. The signals present at a receiver, due to $I$ simultaneous transmissions, is illustrated in Fig. 3.

\subsection{Transmitter model}

Consider the binary data of $\mathrm{WT}_{i}$ as a sequence of mutually independent rectangular pulses of duration $T$ given by

$$
b_{i}(t)=\sum_{l=-\infty}^{\infty} b_{i, l} p_{T}(t-l T),
$$

where $b_{i, l} \in\{+1,-1\}$ for each $l$ and $p_{T}(t)$ represents a unit rectangular pulse in $(0, \mathrm{~T}]$. Suppose that $\psi(t)$ is the chip waveform of duration $T_{c}=T / G_{p}$ such that $G_{p}$ is the processing gain, then the baseband DS spreading signal can be expressed as

$$
x(t)=\sum_{m=-\infty}^{\infty} x_{m} \psi\left(t-m T_{c}\right) .
$$

This spreading sequence is periodic with $x_{m} \in\{+1,-1\}$

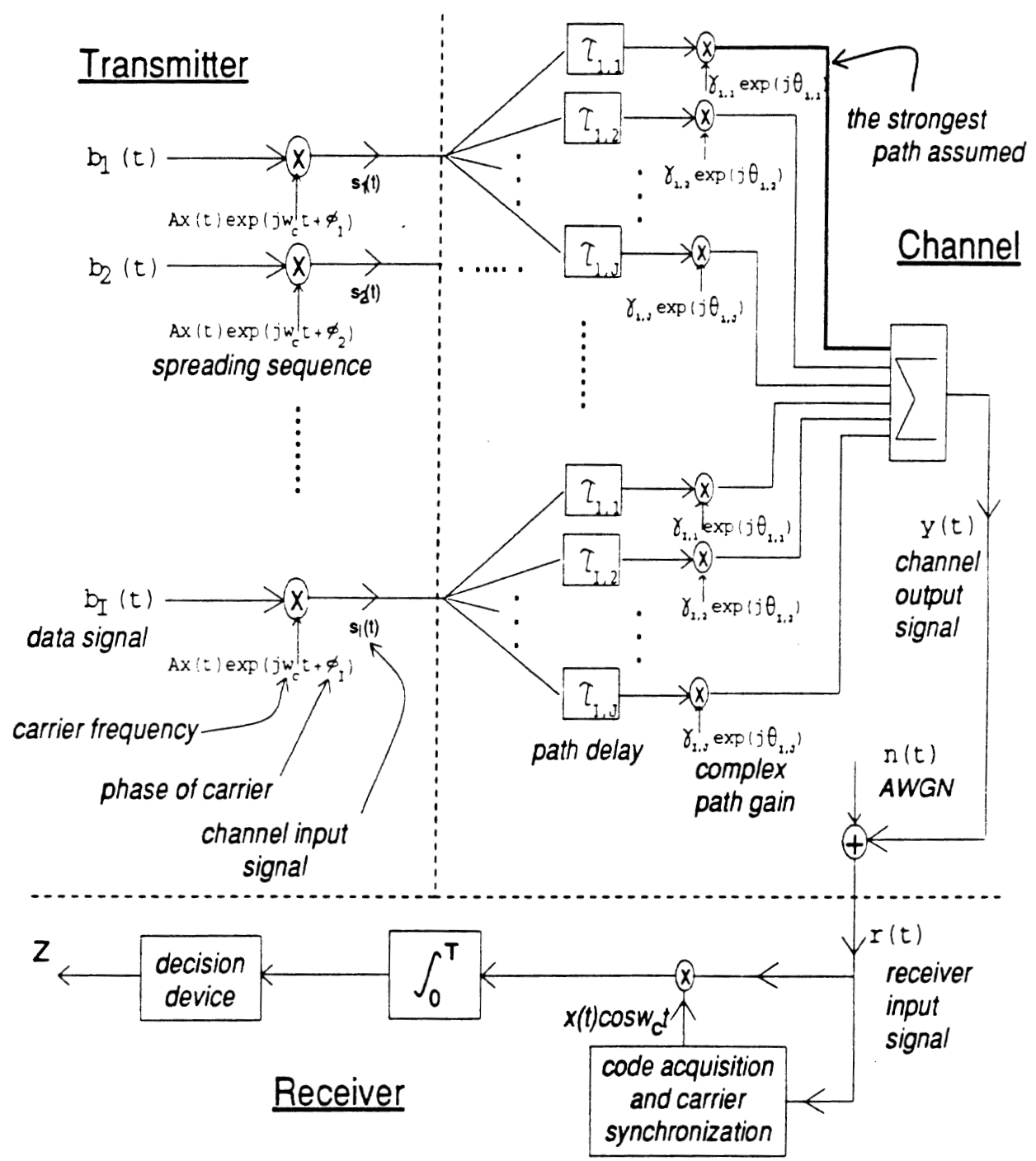

Fig. 3. DS/SS signaling model, with multiaccess and multipath signals at a receiver. 
for each $m$, i.e. $x(t)=x(t-T), x_{m}=x_{m+G_{p}}$. After being spread by $x(t)$ and BPSK-modulated, the data signal $b_{i}(t)$ produces a channel input signal of

$$
s_{i}(t)=\operatorname{Re}\left\{A x(t) b_{i}(t) \exp \left(j \omega_{c} t+\phi_{i}\right)\right\},
$$

where $A$ is the signal amplitude, $\omega_{c}$ the carrier frequency and $\phi_{i}$ the phase of the carrier at $\mathrm{WT}_{i}$.

\subsection{Channel model}

The channel is temporally divided into time slots for slotted Aloha access. It is assumed that WT positions are fixed in each time slot. This assumption is valid if WT's are fixed, or moving very slowly relative to the duration of a time slot. Therefore, the propagation environment for each slot is considered to be static such that the channel exhibits only frequency-selective fading but no time selectivity, i.e., no Doppler spread. Under such conditions, time delay spread is the only propagative degradation affecting signal transmissions. In addition to each input signal to the channel being attenuated and time-delayed, several multipath replicas of it may arrive at each receiver at different delays after travelling along various reflected paths. Hence in a multiaccess channel, the channel output signal consists of a sum of delayed, phase-shifted and attenuated replicas of various input signals given by

$$
\begin{aligned}
y(t)= & \sum_{i=1}^{I} \sum_{j=1}^{J} \operatorname{Re}\left\{\gamma_{i, j} A x\left(t-\tau_{i, j}\right) b_{i}\left(t-\tau_{i, j}\right)\right. \\
& \left.\times \exp j\left[\omega_{c}\left(t-\tau_{i, j}\right)+\theta_{i, j}+\phi_{i}\right]\right\}
\end{aligned}
$$

when $I$ WT's transmit simultaneously and each transmission produces $J$ scatter paths, each introducing a path delay $\tau$ and a complex path gain with magnitude $\gamma$ and phase shift $\theta$. Due to the different path delays for the various receive signal components, it is not necessary for the transmitters to be tightly synchronized.

\subsection{Receiver model}

Consider a receiver capable of synchronizing to the strongest one of several signals present at its input during acquisition. Assume the direct path of $s_{1}(t)$ produces the strongest signal which is acquired by the receiver. Let all $\tau_{i, j}, \theta_{i, j}, \phi_{i}$ be measured relative to $\tau_{1,1}=0$, $\theta_{1,1}=0$, and $\phi_{1}=0$, and represented by the vectors $\vec{\tau}, \vec{\theta}$, and $\vec{\phi}$, respectively. Further, $\vec{b}=\left(b_{1,-1}, b_{1,0}\right)$. After being corrupted by additive white Gaussian noise $n(t)$ with two-sided spectral density $N_{0} / 2$, the received signal is

$$
\begin{aligned}
r(t)= & A \gamma_{1,1} x(t) b_{1}(t) \cos \left(\omega_{c} t\right) \\
& +A \sum_{j=2}^{J} \gamma_{1, j} x\left(t-\tau_{1, j}\right) b_{1}\left(t-\tau_{1, j}\right) \cos \left(\omega_{c} t+\theta_{1, j}\right) \\
& +A \sum_{i=2}^{I} \sum_{j=2}^{J} \gamma_{i, j} x\left(t-\tau_{i, j}\right) b_{i}\left(t-\tau_{i, j}\right) \\
& \times \cos \left(\omega_{c} t+\theta_{i, j}+\phi_{i}\right)+n(t)
\end{aligned}
$$

Correlation with the synchronized locally generated PN code gives the receiver output

$$
\begin{aligned}
Z= & \int_{0}^{T} r(t) x(t) \cos \left(\omega_{c} t\right) d t \\
= & \frac{1}{2} A T \gamma_{1,1} b_{1,0}+\frac{1}{2} A \sum_{j=2}^{J} \gamma_{1, j}\left[b_{1,-1} R_{x}\left(\tau_{1, j}\right)\right. \\
& \left.+b_{1,0} \hat{R}_{x}\left(\tau_{1, j}\right)\right] \cos \theta_{1, j} \\
& +\frac{1}{2} A \sum_{i=2}^{I} \sum_{j=2}^{J} \gamma_{i, j}\left[b_{i,-1} R_{x}\left(\tau_{i, j}\right)\right. \\
& \left.+b_{i, 0} \hat{R}_{x}\left(\tau_{i, j}\right)\right] \cos \left(\theta_{i, j}+\phi_{i}\right)+\eta \\
= & \frac{1}{2} A T\left[\gamma_{1,1} b_{1,0}+\zeta(\boldsymbol{b}, \tau, \theta, \phi)\right]+\eta
\end{aligned}
$$

where the interference component

$$
\begin{aligned}
\zeta(\boldsymbol{b}, \tau, \theta, \phi)= & \frac{1}{T} \sum_{j=2}^{J} \gamma_{1, j}\left[b_{1,-1} R_{x}\left(\tau_{1, j}\right)\right. \\
& \left.+b_{1,0} \hat{R}_{x}\left(\tau_{1, j}\right)\right] \cos \theta_{1, j} \\
& +\frac{1}{T} \sum_{i=2}^{I} \sum_{j=1}^{J} \gamma_{i, j}\left[b_{i,-1} R_{x}\left(\tau_{i, j}\right)\right. \\
& \left.+b_{i, 0} \hat{R}_{x}\left(\tau_{i, j}\right)\right] \cos \left(\theta_{i, j}+\phi_{i}\right),
\end{aligned}
$$

$R_{x}(\tau)=\int_{0}^{\tau} x(t-\tau) x(t) d t$ is the continuous-time partial autocorrelation function of $x, \hat{R}_{x}(\tau)=R_{x}(T-\tau)$, and $\eta$ is a zero mean Gaussian random variable with variance $\frac{1}{4} N_{0} T$. One of the assumptions in obtaining (6) is that $\tau_{i, j} \in[0, T]$ which is generally appropriate in an indoor environment. Consequently the interference component in (7) depends on $b_{i, l}$ only for $l=0,-1$ since $b_{i}(t-\tau)=b_{i,-1}$ when $0 \leqslant t<\tau$ and $b_{i}(t-\tau)=b_{i, 0}$ when $\tau \leqslant t<T$.

\subsection{Correlation functions}

In the presence of multipath and multiaccess interference,

$$
\begin{aligned}
R_{x}\left(\tau_{i, j}\right)= & C_{x}\left(G_{p}-l\right) \hat{R}_{\psi}\left(\tau_{i, j}-l T_{c}\right) \\
& +C_{x}\left(G_{p}-l-1\right) R_{\psi}\left(\tau_{i, j}-l T_{c}\right),
\end{aligned}
$$

where $l=\left\lfloor\frac{\tau_{i, j}}{T_{c}}\right\rfloor, R_{\psi}(s)=s, \quad \hat{R}_{\psi}(s)=T_{c}-s, C_{x}$ is the aperiodic autocorrelation function of $x$ and $G_{p}$ is the number of chips per data symbol. Now with (8), (7) can be written as

$$
\begin{aligned}
\varsigma(\boldsymbol{b}, \tau, \theta, \phi)= & \sum_{j=2}^{J} \gamma_{1, j} I\left(\boldsymbol{b}, \tau_{1, j}, \theta_{1, j}, \phi_{1}\right) \\
& +\sum_{i=2}^{I} \sum_{j=1}^{J} \gamma_{i, j} I\left(\boldsymbol{b}, \tau_{i, j}, \theta_{i, j}, \phi_{i}\right)
\end{aligned}
$$

where 


$$
\begin{aligned}
& I\left(\boldsymbol{b}, \tau_{i, j}, \theta_{i, j}, \phi_{i}\right) \\
& =\left\{\begin{array}{l}
\cos \left(\theta_{i, j}+\phi_{i}\right) \frac{b_{i, 0}}{T}\left\{\theta _ { x } ( l ) \left[(l+1) T_{c}\right.\right. \\
\left.\left.-\tau_{i, j}\right]+\theta_{x}(l+1)\left(\tau_{i, j}-l T_{c}\right)\right\}, \quad b_{i, 0}=b_{i,-1}, \\
\cos \left(\theta_{i, j}+\phi_{i}\right) \frac{b_{i, 0}}{T}\left\{\hat { \theta } _ { x } ( l ) \left[(l+1) T_{c}\right.\right. \\
\left.\left.-\tau_{i, j}\right]+\hat{\theta}_{x}(l+1)\left(\tau_{i, j}-l T_{c}\right)\right\}, \quad b_{i, 0} \neq b_{i,-1},
\end{array}\right.
\end{aligned}
$$

and $\theta_{x}$ and $\hat{\theta}_{x}$ are, respectively, the even and odd autocorrelation functions of $x$. M-sequences are well known to have $\theta_{x}(l)=\hat{\theta}_{x}(l)=G_{p}$ if $l \bmod G_{p}=0$, and $\theta_{x}(l)=-1$ if $l \bmod G_{p} \neq 0$, but $\hat{\theta}_{x}(l)$ varies with $l$. In particular, the odd correlation function $\hat{M}(x)=\left\{\left|\hat{\theta}_{x}(l)\right|: 0 \leqslant l \leqslant G_{p}\right\}$ $\geqslant \max \left\{\left|\theta_{x}(l)\right|: 0 \leqslant l \leqslant G_{p}\right\}$. [16] gives values to $\hat{M}(x)$ for different $G_{p}$.

\subsection{Frame capturing probability}

Since (9) still contains many complex correlation functions, we use the method developed in [17] to bound (10) as

$$
\begin{aligned}
&\left|I\left(\boldsymbol{b}, \tau_{i, j}, \theta_{i, j}, \phi_{i}\right)\right| \leqslant \frac{1}{T} \max \left\{\theta_{x}(l), \theta_{x}(l+1),\right. \\
&\left.\hat{\theta}_{x}(l), \hat{\theta}_{x}(l+1)\right\} \\
&=\frac{1}{G_{p}} \max \left\{\hat{\theta}_{x}(l), \hat{\theta}_{x}(l+1)\right\} .
\end{aligned}
$$

Hence, the upper bound derived for $\varsigma(\boldsymbol{b}, \tau, \theta, \phi)$ becomes

$$
\begin{aligned}
|\varsigma(\boldsymbol{b}, \tau, \theta, \phi)| \leqslant & \sum_{j=2}^{J} \frac{\gamma_{1, j}}{G_{p}} \max \left\{\hat{\theta}_{x}(l), \hat{\theta}_{x}(l+1)\right\} \\
& +\sum_{i=2}^{I} \sum_{j=1}^{J} \frac{\gamma_{i, j}}{G_{p}} \max \left\{\hat{\theta}_{x}(l), \hat{\theta}_{x}(l+1)\right\} .
\end{aligned}
$$

Assuming that the receiver decision is based on the usual comparison of the de-spread signal $Z$ with a zero threshold, and $P\left(b_{1,0}=1\right)=P\left(b_{1,0}=-1\right)$, from (6) and (12) we obtain an easily evaluated upper bound for the bit-error probability,

$$
\begin{aligned}
& P_{e}(\boldsymbol{b}, \tau, \theta, \phi)=Q\left(\frac{\gamma_{1,1}-\varsigma|\boldsymbol{b}, \tau, \theta, \phi|}{\frac{2}{A T} \sqrt{\operatorname{var} \eta}}\right) \\
& \leqslant Q\left(\sqrt { \frac { 2 E _ { b } } { N _ { 0 } } } \left[\gamma_{1,1}-\frac{1}{G_{p}} \sum_{j=2}^{J} \gamma_{1, j} \max \left\{\hat{\theta}_{x}(l), \hat{\theta}_{x}(l+1)\right\}\right.\right. \\
& \left.\left.-\frac{1}{G_{p}} \sum_{i=2}^{I} \sum_{j=1}^{J} \gamma_{i, j} \max \left\{\hat{\theta}_{x}(l), \hat{\theta}_{x}(l+1)\right\}\right]\right),
\end{aligned}
$$

where $Q(x)=(1 / \sqrt{2 \pi}) \int_{x}^{\infty} e^{-y^{2} / 2} d y$.

We define the frame capturing probability at a receiver as the probability of successfully receiving one of several colliding frames with no bit errors. Suppose that the $I$ simultaneously transmitting WT's are located at $\boldsymbol{t}_{1}$, $\boldsymbol{t}_{2}, \cdots, \boldsymbol{t}_{I}$ and every frame has a fixed length of $B$ bits. Assume that the bit-error probability for every bit is the same and independent of each other. The frame capturing probability for a receiver located at $\boldsymbol{r}$ is

$$
q_{I}=\left[1-P_{e}(\bar{b}, \bar{\tau}, \bar{\theta}, \bar{\phi})\right]^{B}
$$

where $P_{e}$ is given in (13) and depends on the locations of both the receiver and the transmitters. Given a fixed set of receiver locations, the expected value of this probability for each receiver, with respect to the random transmitter locations, can be expressed as $\bar{q}(\boldsymbol{r})$.

\subsection{Receiver capturing probability}

From (14) we can derive the receiver capturing probability at a location $\vec{t}$ which is the probability that if a frame is transmitted at this location, this frame is captured by at least one receiver. Since signals arrive at different receivers via different paths with independent complex path gains and are corrupted by independent Gaussian noise, we assume independent frame reception probability at different receivers. If $M$ receivers are located at $\vec{r}_{1}, \vec{r}_{2}, \cdots, \vec{r}_{M}$ then the receiver capturing probability at $\vec{t}$ is given by

$$
p\left(\boldsymbol{t}, \boldsymbol{r}_{1}, \boldsymbol{r}_{2}, \cdots, \boldsymbol{r}_{M}\right)=1-\prod_{k \in R}\left[1-\bar{q}\left(\boldsymbol{t}, \boldsymbol{r}_{k}\right)\right]
$$

where $R$ is the set of all receivers, and $\bar{q}(\boldsymbol{t}, \boldsymbol{r})$ is similar to $\bar{q}(\boldsymbol{r})$ above with the exception the averaging is over a random set of transmitters given that a transmitter is located at $\boldsymbol{t}$, and conditioned on the intended transmitter being closer to the receiver than any other active transmitters. This condition ensures that the intended transmitter captures the receiver.

\section{Uplink performance}

The throughput and delay performance of the uplink radio channel between the WT's and the distributed RB's is evaluated in this section. The throughput achieved at a receiver is determined by the number of interfering transmitters within range of the receiver. Since the number of WT's within range of each RB is generally much larger than the number of RB's within range of each WT, the uplink receiver is potentially subject to a much larger number of multiaccess and multipath interference signals, compared to the down link receiver, i.e, the uplink performance should lowerbound the downlink performance under heavy traffic conditions.

We apply the DS/SS signaling model in the last section to a slotted Aloha network with multiple receivers. Given the locations of the transmitters and receivers, (13)-(15) may be employed to analytically obtain the probability that a frame transmitted by a specific WT is 
captured by the receiver in at least one RB. The system's expected channel throughput $S$ and expected packet waiting time $w_{t}$ can be solved by applying this probability to a discrete Markov Chain model similar to [18]. Although the original model is for frame reception with a single receiver, it is easily generalized for the multiple receiver case. However, the averaging of $p\left(\boldsymbol{t}, \boldsymbol{r}_{1}, \boldsymbol{r}_{2}, \cdots\right.$, $\boldsymbol{r}_{M}$ ) with respect to the spatial distribution of WT's require enormous computational efforts, rendering the numerical computation of $S$ and $w_{t}$ impractical. Hence, we use simulations to obtain results for these measures. In the single receiver case, however, analytical results for channel throughput have been obtained to corroborate with the simulation results.

\subsection{Parameters of simulation model}

We consider a WLAN operating inside a $40 \times 40 \mathrm{~m}^{2}$ room, in which $N=100$ WT's are uniformly distributed, and $M$ receivers are located at fixed positions. The room is divided into a grid of 100 squares of $4 \times 4 \mathrm{~m}^{2}$, and a WT is located at the centre of each square. The WT's transmit fixed length frames of $B=1024$ bits to the RB's by means of slotted Aloha multiple access. A time slot is set to 1026 bits with the extra 2 bits as guard bits, allowing the transmitter to be very loosely synchronized. We assume ACKs and NAKs in the return channel (downlink) have no propagation delay and are never lost. We also assume that each idle WT originates a new packet and transmit the resulting frame in the next slot with probability $p_{0}$ and each backlogged WT retransmits in the next slot with probability $p_{r}$ until the frame transmission is successful. A WT is backlogged if its frame transmission is unsuccessful due to multipath or multiaccess interference, or channel noise. In the simulations, the input traffic intensity is varied by using different values for $p_{0}$ whereas a constant value $p_{r}=0.025$ is employed.

For simplicity, we assume that walls are reflective but furniture is not, local scattering is insignificant, the RB's are located below the ceiling so that a direct path is always present between a WT and any RB, and directional antennae are employed which generate and pick up reflections only off the walls but not the ceiling or the floor. Therefore, each transmission from a WT results in one direct path and four multipath signals, the latter due to reflections off the walls. We assume all transmissions have equal power such that the receive bit-energy to noise density ratio at the centre of the room is $E_{b} / N_{o}=10 \mathrm{~dB}$ for a signal transmitted at any corner of the room, in the absence of multiaccess and multipath interference. This ensures sufficient signal energy to overcome thermal noise at most receivers. Each signal is then attenuated using the inverse fourth power of the distance between a receiver-terminal pair over the respective path [19]. Transmissions employ DS/SS signaling as discussed previously, with a short chip duration equiva- lent to $1 \mathrm{~m}$ (i.e., a high chip rate of $300 \mathrm{Mchips} / \mathrm{s}$ ) to combat multipath, i.e., the receiver is able to suppress all multipath signals for which the path length is at least $1 \mathrm{~m}$ longer than the direct path. A relatively high processing gain, $G_{p}=255$, is employed.

The simulation is performed on a slot-by-slot basis, with the set of transmitting WT's in each slot determined by the new frame transmission probability $p_{0}$ for idle WT's, the retransmission probability $p_{r}$ for backlogged WT's. The frame capture probability at each receiver, given by (14), is then calculated for the set of transmitting WT's in the slot, given that the frame transmitted by the active WT closest to the receiver is the target for capture. The channel throughput $S$ is the average number of different frames captured by the receivers in each slot, determined from the relative positions of the set of active WT's and the receivers. The average packet waiting time $w_{t}$ is the average number of slots between the generation of the new packet and the subsequent start of successful transmission of the corresponding frame. These performance statistics are obtained for each set of $M$ and $N p_{0}$ values by running the simulation over approximately 20,000 slots.

\subsection{Results and discussions}

Figs. 4 and 5 show the curves of channel throughput $S$ and average packet waiting time $w_{t}$ respectively, vs. $N p_{0}$ for different values of receiver population $M$. Due to the capture effect of DS/SS signaling, higher maximum throughputs are obtained in Fig. 4, compared to the theoretical maximum throughput of $1 / e$ for conventional slotted Aloha channels, even in the single receiver

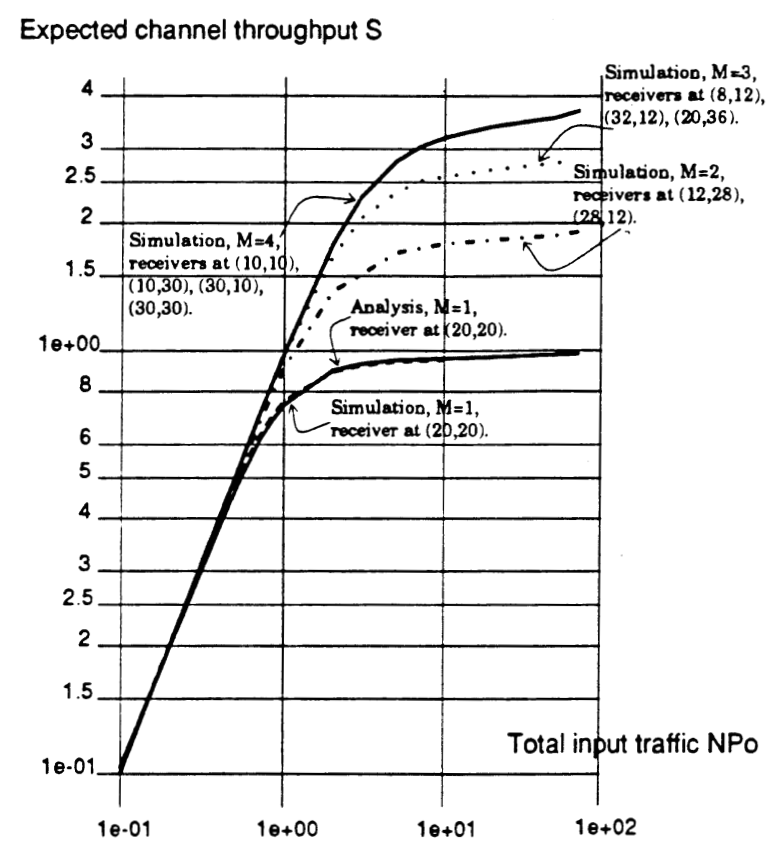

Fig. 4. Throughput versus traffic intensity for uplink channel. 


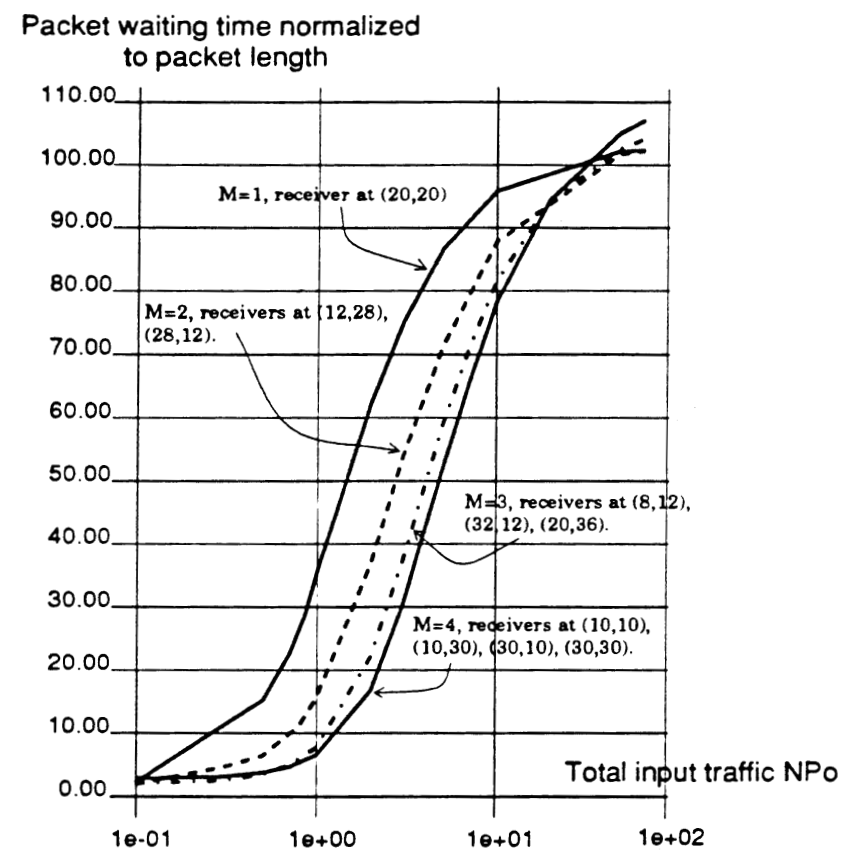

Fig. 5. Average packet waiting time versus traffic intensity for uplink channel.

$(M=1)$ case. Furthermore, as input traffic increases, the channel throughput increases and then stabilizes at its maximum value in Fig. 4, while the average packet waiting time increases and then stabilizes at a finite value in Fig. 5. This is in contrast to conventional finite population slotted Aloha channels which exhibit bi-stable behavior [20] and tend to a low throughput and high delay under heavy traffic. This shows that the multiaccess operation of the DS/SS slotted Aloha channel is mono-stable, a highly desirable property for random access channels. Fig. 4 shows that the maximum throughput value approaches $M$, i.e., under heavy traffic $\left(N p_{0} \gg 1\right)$, the utilization of each receiver approaches $100 \%$. This is due to the fact that under heavy traffic, each receiver is usually captured by a different transmission generated by a WT close to that receiver, so that all receivers are utilized efficiently. However, note that as traffic increases, channel throughput reaches the maximum value at a lower $N p_{0}$ value for smaller $M$ values. This is due to duplicate frame receptions at multiple receivers, which results in a relative decrease in throughput per receiver at medium traffic levels. At such traffic levels, there are more than one access per slot on average, but one transmission may be received in duplication at more than one receivers while another transmission may fail to capture any receiver. While frame duplication results in a penalty in throughput, it results in reduced packet waiting time for larger $M$ values, as shown in Fig. 5. At light traffic level, where on average there is less than one access per slot, a transmission is usually not in competition with others, such that the same throughput level is achieved for all values of $M$. Note that these results are due to the rather benign propagation envi- ronment employed in the model. In a more realistic environment where shadowing of signals occur due to placement of furniture and movements of people, the multiple receiver case should give better channel throughput than the single receiver case, under all traffic levels. However, it is difficult to model a realistic propagation environment in general terms.

Since the DS/SS receiver synchronizes to the strongest signal received, we need to investigate the receiver capturing probability at different locations of the room to see if the near-far effect significantly affects the performance of some WT's. The near-far effect occurs due to the use of equal transmission power by all WT's, resulting in an advantage in receiver capturing for a WT close to a receiver, relative to another WT farther away from the receiver. For this purpose, the room is divided into grids by squares of $4 \times 4 \mathrm{~m}^{2}$ as before. The receiver capturing probability $p\left(\boldsymbol{t}, \boldsymbol{r}_{1}, \boldsymbol{r}_{2}, \cdots, \boldsymbol{r}_{M}\right)$ for an active WT located at the centre of a specific square at coordinate $t$ (with other active WT's randomly distributed over the room) is obtained in simulations and plotted with respect to the WT location in a family of three dimensional curves in Fig. 6 for different values of $M$ with specified receiver locations, and input intensity $N p_{0}$. For $N p_{0}=0.2$ packets $/$ slot as in Fig. 6(i), almost every grid points have similar receiver capturing probabilities, except those at the corners of the room, even for $M=2$ in (a). Uniformity of performance improves with increasing $M$ values. The near-far effect in this case is small because when the traffic is light, there is little multiaccess interference between WT's farther away from a receiver and WT's closer to a receiver. However, as the input intensity increases to 2 packets/slot in Fig. 6(ii) and 5 packets/slot in Fig. 6(iii), substantial variations in receiver capturing probabilities occur for different locations in the room, even with $M=4$. This is because WT's close to the receivers generate enough traffic to capture the receivers most of the time, leaving WT's far away from the receivers with reduced performance. However, it is apparent that increasing the number of receivers reduces these performance variations and eases the impact of the near-far effect, especially under heavy traffic conditions.

In DS/SS systems employing the centralized cellular architecture with a single receiving site per cell, the near far effect is effectively eliminated by the use of power control, such that all WT's in a cell transmit with different power levels which generate equal received signal power at the base site. However, in the distributed, multi-receiver architecture under consideration here, there is no centralized base site. In this case, many power control strategies could be formulated, by adjusting the transmit power of each WT with respect to its distance to (i) the closest RB, (ii) the second closest RB, (iii) the third closest RB, and so on. Our research [21] has shown that these strategies are effective in combating the near-far effect in reducing the performance variations at different 
a) $M=2$ at $(12,28),(28,12)$ :
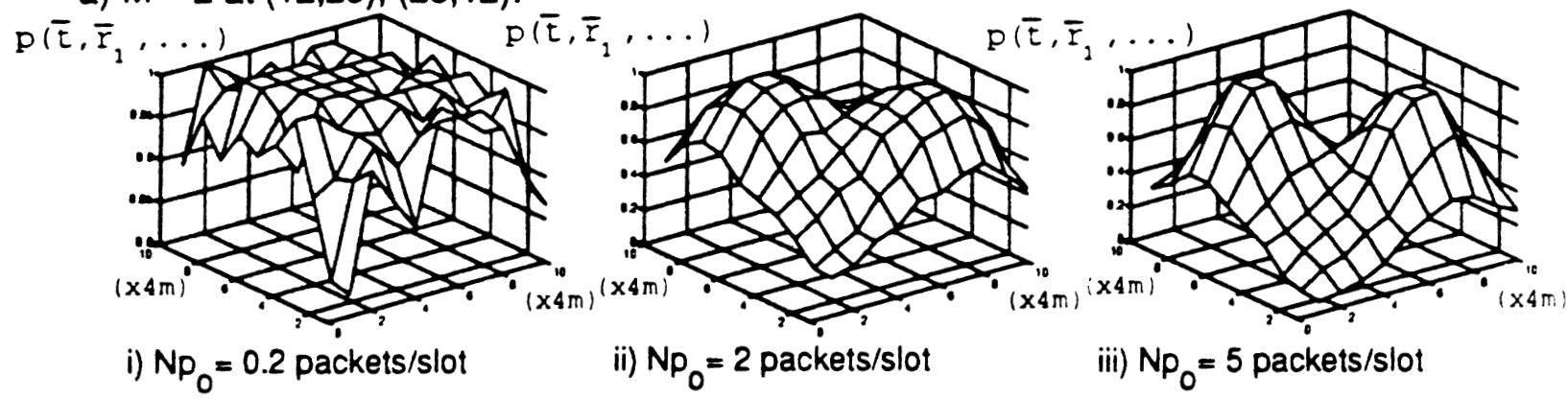

ii) $N p_{0}=2$ packets/slot

iii) $N p_{0}=5$ packets/siot

b) $M=3$ at $(8,12),(32,12),(20,36)$ :

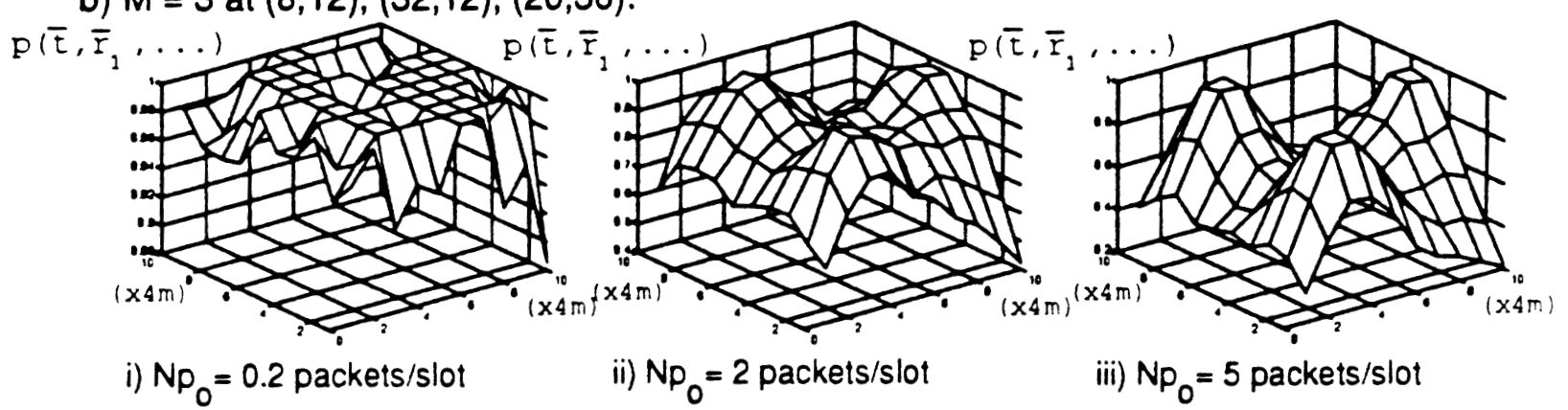

c) $M=4$ at $(10,10),(10,30),(30,10),(30,30)$ :
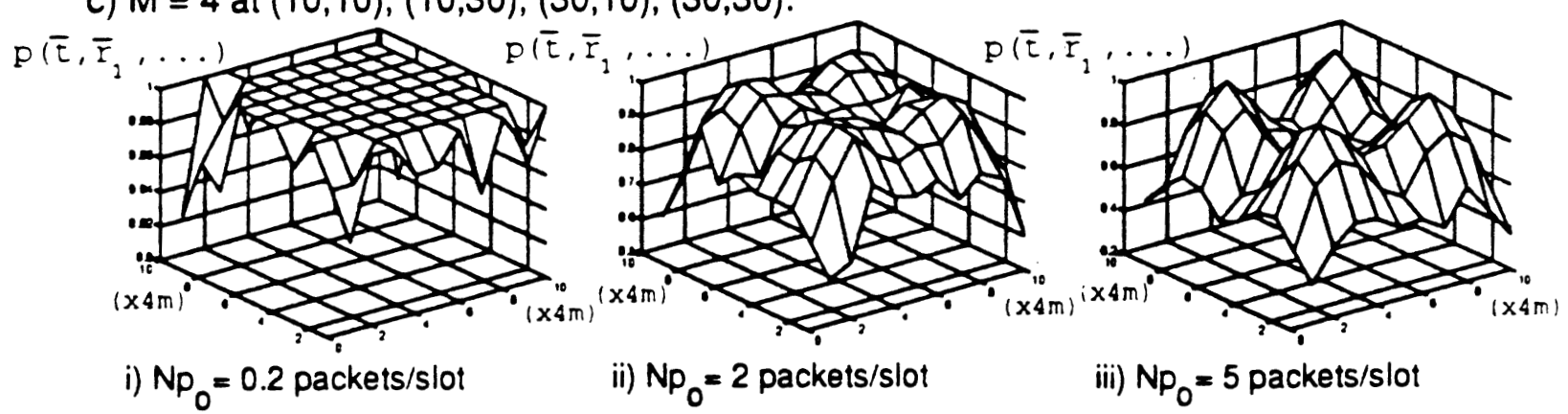

Fig. 6. Three dimensional plots of receiver capture probability at different locations in a $40 \times 40 \mathrm{~m}^{2}$ room

WT locations, but at the expense of reduced average throughput for the entire WT population due to increase in overall multiaccess interference.

\section{Conclusions}

A distributed WLAN architecture for internetworking WT's to a backbone LAN segment via multiple RB's has been presented. Multiple radio links between each WT and the RB's increase the probability of successful reception in the presence of propagation effects. Multipath and multiaccess interference in the wireless channel is further reduced by means of DS/SS signaling. The capture effect of spread-spectrum signaling enables the use of a simple random access protocol. The bridging algorithm in the RB's are based on self-learning routing.
The routing table dynamically adjusts to the changing network connectivity, while the frame forwarding algorithm prevents multiple copies of each frame from being forwarded over the LAN segment. The proposed architecture is deemed to have many attractive properties. In particular, the management of terminal mobility is very much simplified. Simulation results have been presented to quantify the performance of the uplink wireless channel. The system is shown to have high channel throughput capacity. Under heavy traffic, the channel throughput can be maintained at a high level approaching $100 \%$ receiver utilization, while packet waiting time remains finite. These are the contributions of the capture effect. Moreover, by increasing the number of receivers, both the channel throughput and packet waiting time are improved, and the adversity of the near-far effect is reduced. 


\section{References}

[1] K. Pahlavan, Wireless interoffice networks, ACM Trans. Office Inf. Systems 6 (1988) 277-302.

[2] F. Tobagi, Multiaccess protocols in packet communication systems, IEEE Trans. Commun. 28 (1980) 468-488

[3] G. Varghese and R. Perlman, Transparent interconnection of incompatible local area networks using bridges, IEEE J. Sel Areas Commun., 8(1990) 42-48.

[4] D. Buchholz et al., Wireless in-building network architecture and protocols, IEEE Network Mag. 5(1991) 31-38

[5] J.E. Mitzlaff, Radio propagation and anti-multipath techniques in the WIN environment, IEEE Network Mag. 5(1991) 21-26.

[6] K.S. Gilhousen, I.M. Jacob, R. Padovani, A.J. Viterbi, L.A. Weaver Jr. and C.E. Wheatley III, On the capacity of a cellular CDMA system, IEEE Trans. Veh. Techn. 40 (1991) 303312.

[7] R.L. Pickholtz, L.B. Milstein and D.L. Schilling, Spread spectrum for mobile communications, IEEE Trans. Veh. Techn. 40 (1991) 313-322.

[8] M. Serizawa, A radio access scheme for CSMA/CD LAN, Proc. IEEE Int. Conf. on Communication, Boston, MA (1989) pp. 477481

[9] C.M. Chang, Multisite throughput of a mobile data radio link, IEEE Trans. Veh. Techn. 39 (1990) 190-204.

[10] C. Lau and C. Leung, Capture models for mobile packet radio networks, IEEE Trans. Commun. 40 (1992) 917-925.

[11] B. Ramamurthi, A.A.M. Saleh and D.J. Goodman, Perfectcapture ALOHA for local radio communications, IEEE J. Sel. Areas Commun. 5(1987) 806-814.

[12] K. Sakakibara, Performance approximation of a multi-base station slotted ALOHA for wireless LAN, IEEE Trans. Veh. Techn. 41 (1992) 448-454

[13] R.C. Dixon, Spread Spectrum Systems (Wiley, New York, 1976).

[14] A. Santamaria and F.J. Lopez-Hernandez (eds.), Wireless LAN Systems (Artech House, Boston, MA, 1994) ch. 1.

[15] D.V. Sarwate and M.B. Pursley, Crosscorrelation properties of pseudorandom and related sequences, Proc. IEEE 68 (1980) 593619

[16] M.B. Pursley and H.F.A. Roefs, Numerical evaluation of correlation parameters for opotimal phases of binary shiftregister sequences, IEEE Trans. Commun. COM-27 (Oct. 1979).

[17] M.B. Pursley, Effects of specular multipath fading on spread spectrum communications, in: New Concepts in Multi-user Communication (Sijthoff and Noordhoff, 1980) pp. 481-505.
[18] C. Namislo, Analysis of mobile radio slotted ALOHA network, IEEE J. Sel. Areas Commun. 2(1984) 583-588

[19] M. Kavehrad and G.E. Bodeep, Design and experimental results for a direct-sequence spread-spectrum radio using differential phase-shift keying modulation for indoor, wireless communications, IEEE J. Sel. Areas Commun. 5 (1987) 815-823.

[20] A.B. Carleial and M.E. Hellman, Bi-stable behavior of slotted Aloha-type systems, IEEE Trans. Commun. 23 (1975) 401-410.

[21] A.W.Y. Au, Multi-receiver performance of slotted aloha access with direct sequence spread-spectrum signaling for wireless inbuilding networks, UBC M.A.Sc. Thesis (1993).

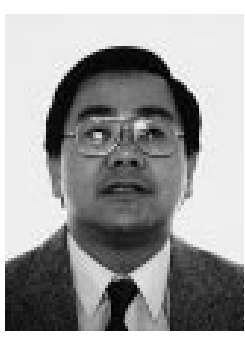

Victor C.M. Leung received the B.A.Sc. (Hons.) degree in electrical engineering from the University of British Columbia (U.B.C.) in 1977, and was awarded the APEBC Gold Medal as the head of the graduating class in the Faculty of Applied Science. He attended graduate school at U.B.C. on a Natural Sciences and Engineering Research Council Postgraduate Scholarship and obtained the $\mathrm{Ph} . \mathrm{D}$. degree in electrical engineering in 1981. From 1981 to 1987, he was a Senior Member of Technical Staff at Microtel Pacific Research Ltd. (now known as MPR Teltech Ltd.), specializing in the planning, design and analysis of satellite communication systems. He also held a part-time position as Visiting Assistant Professor at Simon Fraser University in 1986 and 1987. In 1988, he was a Lecturer in the Department of Electronics at the Chinese University of Hong Kong. He joined the Department of Electrical Engineering at U.B.C. in 1989, where he is an Associate Professor and a member of the Centre for Integrated Computer Systems Research. He is also a project leader in the Canadian Institute for Telecommunications Research, a Network of Centres of Excellence funded by the Canadian Government. His research interests are in the areas of architectural and protocol design and performance analysis for computer and telecommunication networks, with applications in satellite, mobile, personal communications and high speed networks.

E-mail: vleung@ee.ubc.ca

Andrew W.Y. Au. Photograph and biography not available at time of publication. 